\title{
Strategies for Lidar Characterization of Particulates from Point and Area Sources
}

\author{
Michael D. Wojcik*a, Kori D. Moore ${ }^{\mathrm{a}}$, Randal S. Martin ${ }^{\mathrm{b}}$, Jerry Hatfield ${ }^{\mathrm{c}}$ \\ ${ }^{a}$ Energy Dynamics Laboratory, Utah State University, 1695 North Research Park Way, North \\ Logan, UT, USA 84341; \\ ${ }^{b}$ Dept. of Civil and Environmental Engineering, Utah State University, 4110 Old Main Hill, \\ Logan, UT, USA 84322-4110; \\ ${ }^{c}$ National Laboratory for the Agriculture and the Environment, 2110 University Blvd. Ames, \\ IA, USA 50011
}

\begin{abstract}
Use of ground based remote sensing technologies such as scanning lidar systems (light detection and ranging) has gained traction in characterizing ambient aerosols due to some key advantages such as wide area of regard $\left(10 \mathrm{~km}^{2}\right)$, fast response time, high spatial resolution $(<10 \mathrm{~m})$ and high sensitivity. Energy Dynamics Laboratory and Utah State University, in conjunction with the USDA-ARS, has developed a three-wavelength scanning lidar system called Aglite that has been successfully deployed to characterize particle motion, concentration, and size distribution at both point and diffuse area sources in agricultural and industrial settings. A suite of massbased and size distribution point sensors are used to locally calibrate the lidar. Generating meaningful particle size distribution, mass concentration, and emission rate results based on lidar data is dependent on strategic onsite deployment of these point sensors with successful local meteorological measurements. Deployment strategies learned from field use of this entire measurement system over five years include the characterization of local meteorology and its predictability prior to deployment, the placement of point sensors to prevent contamination and overloading, the positioning of the lidar and beam plane to avoid hard target interferences, and the usefulness of photographic and written observational data.
\end{abstract}

Keywords: lidar, PM, OPC, sample strategies, remote sensing, point sampler, calibration

\section{INTRODUCTION}

Historically, ambient particulate concentrations and source emission rates generally have been characterized using point sampler technologies. In recent years particle sampling via remote sensing based instruments has increased; some of the remote sensing techniques that have been employed include light detection and ranging (lidar), open path Fourier transform spectroscopy, and open path laser-based detection. ${ }^{1,2,3}$ Remote sensors have been deployed on land, air and satellite platforms. While often low-cost, point sensors tend to be filter-based, sample a small volume of air, are usually stationary, and have relatively long sample times. Standard, regulatory accepted methods of characterizing and quantifying pollutants in the atmosphere are almost exclusively point sample techniques. On the other hand, remote sensing techniques generally sample large volumes, have short sample times, can be fixed or mobile, and have higher initial instrument and associated maintenance costs. Remote sensors usually require a highly skilled operator, while point sensors can be operated and maintained by technicians with minimal training. Remote sensing techniques require calibration, usually locally, against one or

Remote Sensing for Agriculture, Ecosystems, and Hydrology XII, edited by Christopher M. U. Neale, Antonino Maltese, Proc. of SPIE Vol. 7824, 78240R - (C) 2010 SPIE · CCC code: 0277-786X/10/\$18 - doi: 10.1117/12.865079 
more point sensors. It is important to realize that the above descriptions are generalizations; as a point of fact any specific point or remote sensing method will vary in its characteristics.

Both point and remote sampling methods have advantages and disadvantages, some of which are shared. Theoretically, the advantages of each type can be leveraged and maximized through a combination of point and remote sampling technologies to yield a useful dataset, while at the same time reducing the effects of potential disadvantages from one method that are covered by another. For example, Energy Dynamics Laboratory (EDL) and Utah State University's Environmental Engineering Division, in cooperation with the USDA Agricultural Research Service (ARS), have gathered and developed a suite of both point and remote sensing instruments, collectively called Aglite to characterize and map PM mass concentrations and emissions for various agricultural processes. The central piece of this system is the Aglite lidar, a portable scanning lidar system with a pulsed, three-wavelength laser. A collection of point sensors supports the Aglite lidar; these include several optical particle counters (OPCs), numerous size-fractioning filter-based mass samplers and three different meteorological stations. More than ten field studies conducted over a five year period in agricultural and industrial settings have proven the power and utility of this combination, along with providing the researchers with a greater understanding of maximizing benefits from its application. ${ }^{2,45}$ This paper lists the assembled instruments, briefly describes the role each fills, and provides successful strategies for field deployment and data analysis in general and specifically pertaining to the use of lidar for characterizing aerosol concentrations.

\section{BACKGROUND}

The Aglite measurement system for particulate matter is composed primarily of filter-based portable PM samplers with $\mathrm{PM}_{1}, \mathrm{PM}_{2.5}, \mathrm{PM}_{10}$, and TSP sample configurations, optical particle counters (OPCs), and the Aglite lidar. Supporting equipment and methods used by EDL and its cooperators at Utah State University (USU) and ARS have included meteorological stations, towers instrumented for vertical meteorological characterization, eddy covariance systems, energy balance systems, a particulate organic carbon and elemental carbon analyzer, microscopic analysis of filter catch, ion chromatographic analysis of filter samples, cascade impactors, an aerosol mass spectrometer, and oiled deposition plates. Additionally, the following equipment has been used to characterize gaseous emissions from facilities but will not be discussed herein: passive $\mathrm{NH}_{3}, \mathrm{NO}_{\mathrm{x}}$, and $\mathrm{SO}_{\mathrm{x}}$ samplers; an ambient chemiluminescent $\mathrm{NO}_{\mathrm{x}}$ analyzer; a pulsed fluorescence $\mathrm{SO}_{\mathrm{x}}$ analyzer; a UV photometric ozone analyzer; open-path Fourier transform spectrometer; and an annular denuder system for gaseous anions and cations.

A typical PM sample layout is composed of an array of sample locations surrounding the facility/area/activity of interest, with the majority of locations on the dominantly downwind side of the field. An OPC and multiple portable PM samplers, each with a different particle fractionation configuration, are collocated in order to provide particle number count, particle size distribution, and mass concentrations at one location. OPCs are set to sum particle counts by size for nominal 20 second sample periods throughout the test, while the portable PM samplers provide a period-averaged concentration. These PM support instruments are also calibrated relative to each other on-site to prevent instrument-to-instrument bias. OPCs and PM samplers are typically located at heights of $2 \mathrm{~m}$ and $9 \mathrm{~m}$ above ground level to differentiate vertical concentration gradients.

The Aglite lidar is housed in a portable, generator-powered, temperature controlled trailer with the beam directed through the roof and off a mirror that can scan both in azimuth $\left(280^{\circ}\right.$ range $)$ and elevation $\left(-5^{\circ}\right.$ to $\left.+45^{\circ}\right)$. The laser in the lidar is a Nd:YAG laser pulsed at $10 \mathrm{kHz}$ with $6 \mathrm{~W}$ of power among the emitted beams at 1064 $\mathrm{nm}(3 \mathrm{~W}), 532 \mathrm{~nm}(2 \mathrm{~W})$, and $355 \mathrm{~nm}(1 \mathrm{~W})$. Particles in the atmosphere scatter some of the light back toward the trailer, where it is quantified, with the amount of backscatter dependent on aerosol composition and size. Calibration of the lidar to particulate number and mass concentration is performed by holding the beam in place 
for 60-120 seconds immediately adjacent to an elevated, collocated sample site with an OPC and PM filterbased samplers, a procedure called "a stare". This calibration can then be applied in post collection analysis throughout the field of view of the lidar to create particle concentration maps with time. The Aglite lidar has an operable range of $500 \mathrm{~m}$ to $15 \mathrm{~km}$, and is generally located $500-700 \mathrm{~m}$ from the area of interest in a crosswind position that allows for a full field of view of the air volume immediately above, upwind, and downwind of the operation. Within the range of operation, the spatial resolution of the Aglite lidar system is on the order of $6 \mathrm{~m}$. See Marchant et al. for an in-depth description of the lidar system and Zavyalov et al. for a discussion of the lidar calibration procedure. ${ }^{2,4}$ Figure 1 shows the Aglite instrument in its trailer and Figure 2 shows Aglite deployed at a swine finishing facility.

Facility/operation emission rates can be calculated through two methods. First, mass concentrations measured by the point sensors resulting from the source (calculated by subtracting the upwind/background aerosol concentration from downwind concentrations) are coupled with air dispersion modeling (ISCST3, AERMOD) to calculate observed emission rates through inverse modeling. In inverse modeling, the resulting concentrations are known and the emission rate is unknown; an emission rate is assumed and then adjusted to match the modeled concentrations to the measured concentrations. The second method uses the mass-calibrated lidar data. Vertical lidar scans of the upwind and downwind air, combined with a horizontal scan over the area of interest, create a virtual boundary around the source, referred to as a "staple" owing to its visual representation, and allow for the use of a simple mass balance approach to calculate the source emission rate. Measured wind velocity profiles from the on-site meteorological stations provide the volumetric flow. Bingham et al. provide the detailed methodology for deriving emission rates from mass calibrated lidar data. ${ }^{5}$

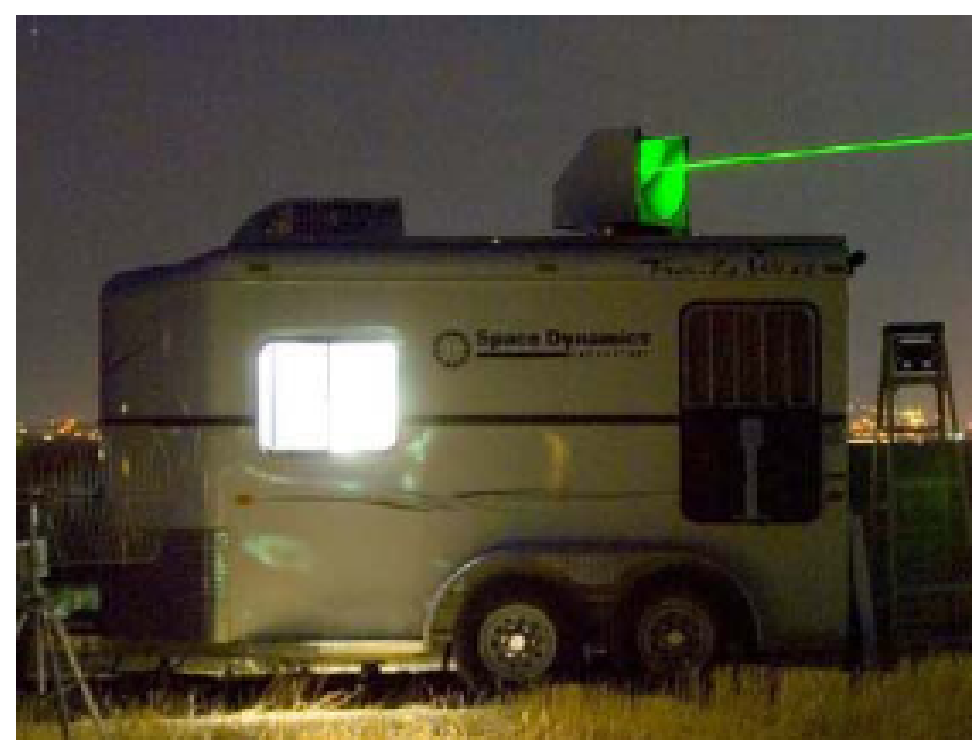

Figure 1. The Aglite instrument in its trailer deployed in an agricultureal location at dusk. 


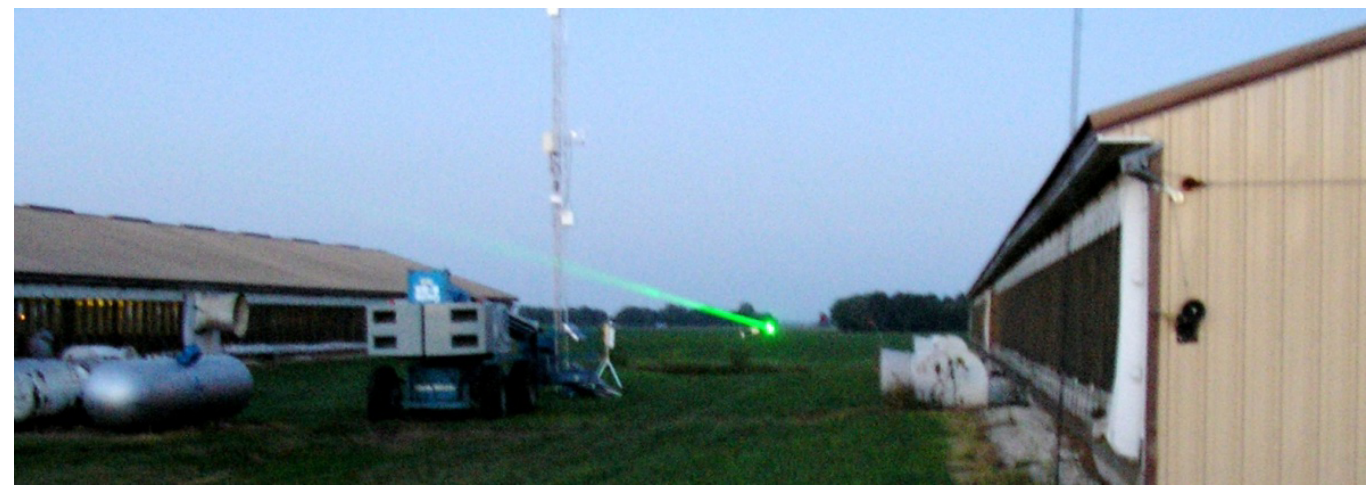

Figure 2. Aglite at a swine finishing facility. The Aglite trailer is in the center of the photo about 1 mile in the distance.

\section{DISCUSSION}

As is often the case with new instrumentation and technologies, the successful operation in the laboratory often is tested and refined through field campaign usage. Since Aglite's maiden deployment in August of 2005 at a swine production facility in Iowa, the system has been used to measure concentrations, emission rates and process fluxes from two dairy production operations, an almond harvesting operation, a cotton ginning operation, a fall tillage sequence, a spring tillage sequence and farm vehicle traffic on unpaved roads. To faithfully determine particle flux resulting from agricultural and industrial processes it is necessary to know both the concentration of particles and the wind speed which drives their transport.

It is difficult to overstate the critical importance of reliable meteorological data during preparation and execution of field measurements. One of the first activities during the early planning phases of any field measurement is to collect as much local meteorological data as possible. One publicly available source is towers/stations supported by the National Weather Service, NOAA, or state/municipal agencies. These data sources are, of course, invaluable but due to their large spatial separation often do not adequately capture the local on-site microclimate at the facility under test. Our solution is to install our own ground station equipped with a cellular phone data uplink and collect at least three weeks of continuous on-site meteorology data immediately prior to deployment. From these data we calculate wind roses and can begin to get a sense of the directional reliability of the wind patterns. We find that a small weather station located on-site allows us to more accurately site out instrumentation than if we relied solely on historical data from the closest established weather station. In addition, when studying intermittent activities, such as agricultural harvest or tillage operations, the knowledge of how well hourly weather predictions match observed conditions is invaluable. This is accomplished by comparing weather predictions downloaded during the pre-sampling deployment of the weather station to measured conditions.

The placement of point sensors during a field measurement is a strong function of the local meteorology and expected source strength. For example, sensors placed too close to the upwind side of a facility may be impacted from the emission source, especially under light and variable wind conditions. In addition it is important to minimize non-source impacts such as vehicle travel on unpaved farm roads or other particulate generating activities on adjacent property. In the undesirable but unavoidable case where external events affect one or more point sensors, the collocation of a point sensor that has high time resolution (e.g. an OPC with a short sample 
time) can be very helpful in identifying potential effects of contamination events on filter-based sampling by inspection of the time-series distribution and concentration data.

Factors that influence the placement of a lidar remote sensor include eye safety, area topography, adequate lineof-sight access to the facility, and clear line-of-sight access to several of the point samplers. Eye-safety is of obvious importance to facility staff, research staff and also to wildlife. Area topography can affect the lidar placement because optical backscatter from hard targets such as trees and distant landscape features (such as mountains as far as 15 miles away) can overload the receiver photodetectors and cause timing errors. In fact, even minor optical interference from telephone or electrical wires can make lidar data unusable, we therefore find that it is best to give a wide berth $(\sim 10 \mathrm{~m})$ to all physical objects. Since the dynamic calibration of our Aglite system depends on regular comparison of the lidar signal to OPC and mass-based point samplers, which are often located on $10 \mathrm{~m}$ tall metal towers, we take great care to make sure that our laser pointing is carefully considered and is free from data artifacts.

In practice, no sample location is ideal and we must work with the site in its existing state; field work in the real world always presents it challenges. Experience has taught us to fill our notebooks with copious amounts of observational data about the site, the daily activities and the processes throughout the day. On a startlingly regular basis, these notes play a critical role when validating and error checking our data. In addition, we have found that photographs of research activities, source activities (both those being studied and those potentially interfering), facility layout, and sample layout have proven invaluable in post-analysis and reporting.

\section{SUMMARY}

While point measurements are the standard methodology for measuring air quality, the use of remote sensing techniques is increasing. Energy Dynamics Laboratory and Utah State University in cooperation with the USDA-ARS, has organized a suite of point samplers to use in conjunction with and as local calibrations for the Aglite lidar, a portable scanning multiwavelength lidar system. The entire composite system provides a basis in traditional sampling methods and, through calibration of the pulsed lidar, is able to extend mass concentration measurement capabilities to larger areas, higher elevations, and with greater time resolution. Deployment strategies learned from field use of this system include the characterization of local meteorology and its predictability prior to deployment, the placement of point sensors to prevent contamination and overloading, the positioning of the lidar and beam plane to avoid hard target interferences, and the usefulness of photographic and written observational data.

\section{ACKNOWLEDGMENTS}

The development of the Aglite system was performed under USDA Agreement number 58-3625-4-121 with Dr. Jerry Hatfield, the Director of the National Laboratory for Agriculture and the Environment in Ames, Iowa, providing valuable direction to the Aglite development team. Any opinions, findings, conclusions, or recommendations herein expressed are those of the authors and do not necessarily reflect the view of the USDA. 


\section{REFERENCES}

1. Arnott, W.P., Schmitt, C., Liu, Y., and Hallett, J. "Droplet size spectra and water-vapor concentration of laboratory water clouds: inversion of Fourier transform infrared (500-5000 $\left.\mathrm{cm}^{-1}\right)$ optical-depth measurement," Applied Optics, 36(21), 5205-5216, (1997).

2. Marchant, C.C.; T.D. Wilkerson; G.E. Bingham; V.V. Zavyalov; J.M. Andersen; C.B. Wright; S.S. Cornelsen; R.S. Martin; P.J. Silva; J.L. Hatfield. "Aglite lidar: A portable elastic lidar system for investigating aerosol and wind motions at or around agricultural production facilities," J. Appl. Remote Sens. 3(1), 033511 (2009) [doi:10.1117/1.3097928].

3. Wang, J., Hiscox, A.L., Miller, D.R., Meyer, T.H., Sammis, T.W., "A comparison of Lagrangian model estimates to light detection and ranging (LIDAR) measurements of dust plumes from field tilling." J. Air \& Waste Manage. Assoc. 59(11) 1370-1378 (2009) [doi:10.3155/1047-3289.59.11.1370].

4. Zavyalov, V.V.; C.C. Marchant; G.E. Bingham; T.D. Wilkerson; J.L. Hatfield; R.S. Martin; P.J. Silva; K.D. Moore; J. Swasey; D.J. Ahlstrom; T.L. Jones. "Aglite lidar: Calibration and retrievals of well characterized aerosols from agricultural operations using a three-wavelength elastic lidar," J. Appl. Remote Sens. 3(1), 033522 (2009) [doi: 10.1117/12.833365].

5. Bingham, G.E.; C.C. Marchant; V.V. Zavyalov; D.J. Ahlstrom; K.D. Moore; D.S. Jones; T.D. Wilkerson; L.E. Hipps; R.S. Martin; J.L. Hatfield; J.H. Prueger; R.L. Pfeiffer. "Lidar based emissions measurement at the whole facility scale: Method and error analysis," J. Appl. Remote Sens. 3(1), 033510 (2009) [doi:10.1117/1.3097919]. 